\title{
INTOXICAÇÃO CÚPRICA ACUMULATIVA EM OVINO: TRATAMENTO E RECUPERAÇÃO (RELATO DE CASO)
}

\author{
ZARO, Débora ${ }^{1}$; \\ MIKHAILENKO, Thayane Santana ${ }^{1}$; \\ LOSS, Daiene Elisa ${ }^{2}$; \\ BUENO, Flávia Umpierre ${ }^{2}$; \\ MELO, Luciano Cavalheiro ${ }^{2}$; \\ BECK, Carlos Afonso de Castro ${ }^{3}$; \\ ROCHA, André Luiz de Araújo ${ }^{3}$; \\ REDIVO, Cristine Bitencourt ${ }^{4}$.
}

Recebido: $20 / 09 / 2017$

Aceito: 01/03/2018

\begin{abstract}
${ }^{1}$ Médicas Veterinárias Residentes, Clínica e Cirurgia de Grandes Animais, Hospital de Clínicas Veterinárias, Faculdade de Veterinária/UFRGS; ${ }^{2}$ Médicos Veterinários e Doutorandos, Programa de Pós-Graduação em Medicina Animal: Equinos/HCV/UFRGS; ${ }^{3}$ Médicos Veterinários, Doutores, Professores do Departamento de Medicina Animal, Faculdade de Veterinária/UFRGS; ${ }^{4}$ Aluna de Graduação em Medicina Veterinária/FAVET/UFRGS.
\end{abstract}

\section{RESUMO}

A intoxicação por cobre é uma das principais causas de mortalidade por intoxicação em ovinos. Os sinais clínicos e anatomopatológicos são similares aos de outras doenças. Assim, vários casos tornam-se inconclusivos quando não são utilizadas técnicas analíticas no diagnóstico. O presente trabalho relata o caso de um carneiro da raça Hampshire Dow que foi encaminhado ao Hospital de Clínicas Veterinárias da Universidade Federal do Rio Grande do Sul com sinais clínicos de intoxicação por cobre. O animal apresentava apatia, inapetência, emagrecimento, dispneia, tosse, secreção nasal, urina e diarreia de coloração escura. O ovino foi adquirido numa feira de exposição de animais e havia permanecido em manejo intensivo recebendo quantidades de concentrado acima da exigência dietética nos dois meses anteriores e durante o evento. Quatro dias após o surgimento dos sinais clínicos o animal foi internado no hospital, foi estabelecida terapia de suporte com fluidoterapia, suplementação de aminoácidos e glicose. Administrou-se sulfato de sódio, molibdato de amônia, adsorvente de toxinas e protetores de mucosa. Após 34 dias de tratamento intensivo o paciente apresentou melhora do quadro clínico e recebeu alta. Baseado no histórico, sensibilidade da espécie, sinais clínicos, resultados dos exames laboratoriais, melhora do quadro clínico e resposta positiva à terapêutica, sugere-se o quadro de intoxicação cúprica acumulativa. Não foi realizada análise histoquímica específica para marcação de cobre. $O$ trabalho consiste em revisão literária, características clínicas e laboratoriais da intoxicação, conduta para o tratamento e diagnóstico, contribuindo, portanto, para o entendimento da doença e redução da ocorrência de novos casos.

Palavras-chave: Cobre. Hemoglobinúria. Hemólise. Diagnóstico. 


\section{INTRODUÇÃO}

A intoxicação por cobre $(\mathrm{Cu})$ é uma causa importante de mortalidade por intoxicação em ovinos, uma das espécies mais sensíveis ao elemento (MIGUEL et al., 2013; RIET-CORREA et al., 2001). A etiopatogenia da doença está associada a menor capacidade de excreção do Cu nesses animais, isso se deve aos baixos teores de metalotioneína, proteína que atua como armazenadora e transportadora desse metal, diminuindo a excreção do cobre no organismo, o qual se acumula no fígado. Os ovinos, em virtude de herança genética, possuem níveis baixos da metaloenzima glutationa peroxidase no sangue, enzima envolvida no transporte do Cu intracelular (RADOSTITS et al., 2000). Segundo Reis et al. (2015) os ovinos apresentam alta sensibilidade ao cobre também por apresentarem baixa capacidade de conjugá-lo à metalotioneína, diminuindo sua excreção por via biliar e ocasionando o acúmulo no fígado.

Os fatores predisponentes relacionados à intoxicação cúprica em ovinos são variados, sendo que o fator racial é um dos mais importantes (ANTONELLI et al., 2010). As raças de carne são mais sensíveis que as raças de lã (RIET-CORREA et al., 2001), essas tendem a absorver e reter mais cobre que as demais, apresentando menor necessidade deste elemento que as raças de lã, as quais utilizam-no para síntese dos folículos pilosos (FERREIRA et al., 2008).

No Rio Grande do Sul (RS) a intoxicação por cobre tem sido diagnosticada principalmente em ovinos preparados para exposições, confinados, e submetidos à alimentação com ração em quantidade acima da exigência dietética (RIBEIRO et al., 2007; RIET-CORREA et al., 2001). É a mais frequente causa de mortalidade no estado, só perdendo para as gastrenterites parasitárias (FERREIRA et al., 2008). Correa (2014), em seu estudo retrospectivo das causas de morte de ovinos diagnosticados no Setor de Patologia Veterinária da Universidade Federal do Rio Grande do Sul, entre os anos 2002 e 2012, verificou que do total de casos 40,43\% eram de doenças infecciosas e parasitárias; $41,66 \%$ eram de intoxicações e toxiinfecções, sendo 6,37\% casos de intoxicação por cobre; $12,34 \%$ eram doenças metabólicas e nutricionais; 5,55\% outros distúrbios, sendo a hemoncose a principal causa de morte de ovinos na pesquisa. A ovinocultura tem crescido expressivamente em algumas regiões do Brasil, porém a maioria dos produtores desconhece as exigências e o manejo nutricional da espécie (MIGUEL et al., 2013). Essa expansão e intensificação nos sistemas de produção 
aumentaram a incidência de diversas enfermidades, desordens nutricionais e metabólicas (ANTONELLI et al., 2010). Fatores estressantes como alterações na dieta, mudança de ambiente, transporte e lactação podem desencadear a enfermidade. A doença crônica ou acumulativa apresenta desenvolvimento lento, porém aparecimento súbito (RIBEIRO, 2011). A intoxicação cúprica acumulativa (ICA) se caracteriza por duas fases: subclínica, em que o $\mathrm{Cu}$ se acumula nos tecidos, principalmente no fígado durante semanas ou meses, e fase aguda ou hemolítica, decorrente da liberação na circulação do $\mathrm{Cu}$ acumulado. Esse acúmulo pode ser primário, pela ingestão excessiva de cobre; ou secundário, por intoxicação crônica fitógena ou intoxicação crônica hepatógena (RIET-CORREA et al., 2001). No momento em que os níveis hepáticos máximos são atingidos, o cobre é liberado na corrente sanguínea causando ruptura de hemácias (RIBEIRO, 2011). Os animais podem adoecer e morrer rapidamente em consequência da anemia hemolítica e nefrose hemoglobinúrica. De acordo com Riet-Correa et al. (2001), raramente os animais sobrevivem ao quadro clínico característico de uma crise hemolítica. Geralmente os animais acometidos são de alto valor econômico, reprodutores com grande potencial zootécnico e mantidos em sistema de criação intensivo, o que torna os prejuízos econômicos bastante elevados (ANTONELLI et al., 2010). O quadro caracteriza-se por depressão, anorexia, dispneia, hemólise, anemia, icterícia, hemoglobinúria, fezes líquidas, fétidas e escuras (CÂMARA et al., 2016; RIBEIRO, 2011).

Assim como o excesso do $\mathrm{Cu}$, a carência do elemento também pode acarretar numa ampla variedade de manifestações clínicas. A suplementação pode ser utilizada em áreas onde os sinais clínicos ou as perdas na produtividade ocasionadas pela carência são constantes. Animais com déficit de $\mathrm{Cu}$ podem apresentar diarreia, redução do peso, anemia, fraturas espontâneas, fragilidade ou despigmentação da lã (RIET-CORREA et al., 2001; SMITH, 2010). De acordo com Bandinelli et al. (2013), o cobre é um microelemento essencial ao organismo por atuar nas células em diferentes processos bioquímicos, como na respiração celular, biossíntese de catecolaminas, metabolização do ferro por enzimas cobre-dependentes, na formação de elastina, colágeno e na produção de melanina. 
Este relato descreve um caso de intoxicação cúprica acumulativa em um carneiro da raça Hampshire Down com recuperação do quadro clínico e sucesso do tratamento.

\section{RELATO DE CASO}

Um carneiro da raça Hampshire Down, com um ano de idade, pesando $105 \mathrm{~kg}$, com sinais clínicos de apatia, perda de apetite, emagrecimento, dispneia, tosse, secreção nasal, urina escura, diarreia de coloração preta e fétida, sem aumento de temperatura retal foi atendido no Hospital de Clínicas Veterinárias (HCV). O animal foi adquirido em uma feira de exposição de animais e havia permanecido em manejo intensivo, quando recebeu quantidades de concentrado acima da exigência dietética durante dois meses antes da exposição e durante o evento. Não foi possível realizar análise da concentração de Cu do concentrado, pois o lote utilizado já havia sido consumido quando o paciente começou a apresentar sinais de intoxicação. De acordo com informações fornecidas pelo proprietário que adquiriu o animal, era ofertado em torno de 4 a $4,5 \mathrm{~kg}$ de alimento concentrado por dia, sendo que o fabricante do produto recomendava a suplementação de 1 a $3 \%$ do peso vivo diariamente, dependendo dos objetivos da criação, da fase de produção e das forragens disponíveis. Após o término da feira, o comprador ajustou a quantidade para $1 \%$ do peso vivo ao dia, ou seja, 1 kg de concentrado ao dia. Não foi possível obter histórico anterior, assim como dados de manejo, formulação e quantidade de concentrado ofertado antes da preparação para a exposição.

O tratamento foi iniciado quatro dias após o aparecimento dos sinais clínicos e dois dias após o ovino apresentar hemoglobinúria e diarreia de coloração preta. Nos primeiros treze dias de internação no hospital o paciente permaneceu 24 horas sob fluidoterapia, recebendo ringer lactato $(\mathrm{RL})$. Iniciou-se com dose de manutenção, $40 \mathrm{ml} / \mathrm{kg} / \mathrm{dia}$, durante três dias, depois foi aumentada para $60 \mathrm{ml} / \mathrm{kg} / \mathrm{dia}$, por mais três dias, após seguiu-se com dose de $70 \mathrm{a}$ $80 \mathrm{ml} / \mathrm{kg} / \mathrm{dia}$. Diariamente realizou-se exame clínico e monitoramento da glicemia. Nos exames clínicos realizados, se observou as mucosas pálidas, sem presença de icterícia, aumento das frequências cardíaca e respiratória e intensa dispneia. Além da terapia de suporte com fluidoterapia, foi feita suplementação de aminoácidos e glicose com Glicopan Energy ${ }^{\circledR}$ (Vetnil, SP, Brasil) conforme dose indicada pelo fabricante de $10 \mathrm{ml}$ por via oral 
(VO), duas vezes ao dia. No período em que o animal apresentou diarreia, administrou-se VO, uma vez ao dia (SID), na dose de $80 \mathrm{ml}$ para cada $100 \mathrm{~kg}$ de peso vivo, um composto a base de carvão vegetal ativado, zeolita, caolim e pectina (Enterex ${ }^{\circledR}$, Vetnil, SP, Brasil), que possui funções de adsorver substâncias tóxicas, ácidos biliares e aflatoxinas B em excesso, formar uma camada protetora sobre a mucosa intestinal e fornecer um ambiente desfavorável para a flora bacterina anormal. Durante 21 dias recebeu $1 \mathrm{~g}$ de sulfato de sódio (SS) e $100 \mathrm{mg}$ de molibdato de amônia (MA), VO, SID. Após 10 dias de fluidoterapia o carneiro apresentou edema subcutâneo. Foi realizado raio-X e ultrassonografia do tórax, não havia presença de alterações. A quantidade de infusão de solução de RL foi diminuída para a dose de $30 \mathrm{ml} / \mathrm{kg} /$ dia, pois a fluidoterapia excessiva pode ter sido a causa do edema. De acordo com Pugh (2005), é fundamental monitorar a hidratação e o teor plasmático de proteína para prevenir a ocorrência de edema. Após 34 dias de tratamento e realização de duas transfusões sanguíneas, foi observada progressiva melhora do quadro clínico e o animal recebeu alta hospitalar.

\section{RESULTADOS E DISCUSSÃO}

Não foi possível realizar análise do concentrado, no entanto, somente os níveis de Cu na ração considerados individualmente, não são bons indicadores como fatores de risco na ICA em ovinos. A quantidade de ração ingerida, outros elementos da dieta, raça e manejo dos animais devem ser considerados (MÖLLERKE; RIBEIRO, 2005).

Na urinálise (Tabela 1), se observou urina com coloração marrom, turva, proteinúria discreta e isostenúria, porém a coleta foi realizada após o início da fluidoterapia. Conforme Ferreira (2013) a densidade específica da urina está diretamente relacionada com a capacidade dos rins do animal em concentrá-la. Em pequenos ruminantes a densidade urinária varia de 1.015 a 1.045. A doença renal pode ser causa de densidade baixa, porém deve ser avaliado com critério, outros fatores tais como fluidoterapia e administração de diuréticos, pois podem alterar a densidade urinária (FERREIRA, 2013). A coloração marrom da urina pode resultar da presença de hemoglobina e/ou mioglobina. Nesse caso, por se tratar de um possível quadro de intoxicação por cobre, se sugere que a coloração escura da urina ao exame físico se deve à presença de hemoglobinúria. A coloração da urina é normalmente 
amarelada, alterações na coloração podem sugerir alterações patológicas (FERREIRA, 2013). Normalmente não há presença de proteína na urina, e a reação positiva para sangue oculto indica presença de eritrócitos ou hemoglobinúria (FERREIRA, 2013).

Tabela 1 - Resultados da urinálise da primeira coleta de urina (21/09/2015) e resultados da última coleta (16/10/2015) realizadas durante o período de internação.

\begin{tabular}{llll}
\hline Urinálise & & $\mathbf{2 1 / 0 9 / 2 0 1 5}$ & $\mathbf{1 6 / 1 0 / 2 0 1 5}$ \\
\hline Coleta & & Micção natural & Mição natural \\
\hline \multirow{4}{*}{ Exame Físico } & Volume: & $10 \mathrm{~mL}$ & $50 \mathrm{~mL}$ \\
& Cor: & Marrom & Amarelo claro \\
& Aspecto: & Turvo & Límpido \\
& Densidade: & 1,012 & 1,010 \\
\hline \multirow{2}{*}{ Exame Químico } & Sangue oculto: & $3+$ & Negativo \\
& Proteínas: & $1+$ & Negativo \\
\hline \multirow{3}{*}{ Exame do Sedimento } & Média de & & \\
& eritrócitos/campo & $<5$ & Não foram observados \\
& (400x): & & \\
\hline
\end{tabular}

As mucosas do ovino estavam pálidas e não apresentavam icterícia. Conforme González e Silva (2006), nos ruminantes, não há grandes aumentos de bilirrubina, mesmo em doenças hepáticas, e considera-se que há icterícia quando os níveis de bilirrubina direta no sangue ultrapassam 2,0 mg/dL. O hematócrito estava abaixo do valor de referência para a espécie (Tabela 2). Quando o valor do hematócrito baixou para $16 \%$ foi realizada a primeira transfusão de sangue com uma bolsa de $450 \mathrm{ml}$. Não houve melhora e após oito dias da primeira transfusão, com hematócrito no valor de 14\%, foi feita a segunda transfusão sanguínea com outra bolsa de $450 \mathrm{ml}$ de sangue. Uma semana depois da segunda transfusão o hematócrito seguia abaixo dos valores de referência para a espécie (15\%), entretanto, não foi possível efetuar uma terceira transfusão, pois o doador foi incompatível na prova de compatibilidade sanguínea. Antes de todas as transfusões, provas de compatibilidade sanguínea foram realizadas com os doadores. Estavam acima do valor considerado normal para a espécie a creatinina e a ureia (Tabela 2). A quantificação laboratorial de cobre sérico foi verificada pelo método de espectrofotometria de absorção atômica e a coleta de sangue para o exame foi realizada no primeiro dia de internamento. O nível sanguíneo de Cu estava dentro do esperado para a espécie: $146 \mu \mathrm{g} / \mathrm{dL}$, sendo os valores de referência de 58 a 160 
$\mu \mathrm{g} / \mathrm{dL}$ (JAIN, 1993; KANEKO et al., 1997). O diagnóstico de ICA pode se realizar medindo as concentrações de cobre no soro, no fígado e no rim. Porém, segundo Smith (2010), as concentrações séricas de cobre não se elevam pouco antes ou durante a crise hemolítica. Animais com concentrações tóxicas de $\mathrm{Cu}$ no fígado podem ter concentrações normais ou deficientes de cobre no soro. Portanto, a medição de cobre sérico não é um método confiável para monitoramento de animais com excesso de cobre no fígado. A ICA ocorre pela deposição gradativa do elemento no fígado, e uma vez que quando se satura a capacidade de armazenamento nesse órgão, o cobre torna-se livre nos hepatócitos. $\mathrm{O}$ acúmulo de $\mathrm{Cu}$ ocorre na mitocôndria e nos lisossomos hepáticos causando lesões progressivas nas organelas dos hepatócitos, degeneração ou necrose (OSWEILER, 1998). Costa (2011) cita que este fato é indicado pela liberação de certas enzimas hepáticas como a gamaglutamiltransferase (GGT), a aspartatoaminotransferase (AST) e a sorbitoldesidrogenase (SDH). Neste caso, o aumento da atividade das enzimas (Tabela 2) aspartatoaminotransferase (AST) e gama glutamiltransferase (GGT) podem indicar degeneração hepática. Almeida et al. (2013), descreve as seguintes alterações histopatológicas nos casos de intoxicação por $\mathrm{Cu}$ em ovinos: necrose individual de hepatócitos da região centrolobular, tumefação e vacuolização de hepatócitos nas regiões mediozonais, e em alguns casos, periportais. Ribeiro (2011) aponta aumento das enzimas AST e CPK (creatinofosfoquinase), e diminuição no valor do hematócrito como as principais alterações nos perfis sérico e hemático nos casos de intoxicação por cobre, alterações verificadas neste caso (Tabela 2 ).

Conforme Antonelli et al. (2010) e Radostits et al. (2000), a administração oral de SS, MA, caolim e pectina reduz a absorção do cobre dos tecidos e aumenta a excreção fecal. O MA atua promovendo a quelação do $\mathrm{Cu}$ remanescente disponível no trato gastrointestinal. Estudos anteriores confirmam a administração de tetratiomolibdato de amônio como a melhor opção terapêutica para o tratamento da ICA, pois esta molécula liga-se firmemente ao $\mathrm{Cu}$, diminuindo seus níveis plasmáticos e os marcadores de lesão hepática, assim como interrompendo a hemólise. Porém, a dificuldade de aquisição apresenta-se como importante entrave no seu uso terapêutico em surtos de ICA (CÂMARA et al., 2016). 
As interações entre o $\mathrm{Cu}$, molibdênio (Mo) e enxofre (S) na nutrição dos ruminantes são únicas em seus efeitos na saúde e produção animal (RADOSTITS et al., 2000). O Mo e o S podem combinar-se dentro do rúmen, formando os compostos denominados tiomolibdatos, os quais podem ligar-se fortemente ao $\mathrm{Cu}$, impossibilitando-o de ser absorvido e diminuindo o armazenamento tecidual. O ferro (Fe) também interfere na absorção do Cu. Dessa forma, a frequência de intoxicação cúprica é maior naqueles animais que receberam altos teores de $\mathrm{Cu}$ e baixos de Mo, S e Fe (FERREIRA et al., 2008). Muitos alimentos concentrados, de alto teor energético são razoavelmente ricos em Cu e pobres em Mo, S e Fe, levando à alta disponibilidade do $\mathrm{Cu}$.

Em relação ao diagnóstico diferencial, as seguintes enfermidades devem ser consideradas: leptospirose, piroplasmose e hemoglobinúria bacilar, entretanto essas doenças cursam com quadro febril (ANTONELLI et al., 2010; RIBEIRO, 2011). No RS, o diagnóstico diferencial deve incluir, também, enfermidades que cursem com lesões hepáticas similares à intoxicação por $\mathrm{Cu}$, como: intoxicação pelas plantas hepatotóxicas Xanthium spp., Cestrum intermedium, Cestrum parqui, Trema micrantha, Dodonaea viscosa e pelas larvas de Perreya flavipes (BANDINELLI et al., 2013). De acordo com Neto (2014), a conduta diagnóstica correta deve incluir, além dos achados anatomopatológicos, a avaliação do tecido hepático com a coloração de Ulzmann, para a confirmação do acúmulo de cobre hepático, além da realização de dosagens do mineral no fígado e nos rins. Para Radostits (2000), a biópsia hepática é uma técnica adequada para o diagnóstico, sendo de grande valor na detecção de intoxicação acumulativa pelo $\mathrm{Cu}$, uma vez que os níveis de $\mathrm{Cu}$ no sangue não se elevam até a ocorrência da crise hemolítica logo antes da morte. No presente caso, não foi realizada biópsia hepática em atenção ao proprietário, que demonstrou preocupação com a manipulação e o possível estresse gerado ao animal em decorrência de tal procedimento, já que o paciente se apresentava extremamente debilitado, principalmente no início do tratamento, e também a fim de evitar aumento nos custos do tratamento.

Após 34 dias de tratamento, a creatinina e o hematócrito se aproximaram dos valores normais para a espécie (Tabela 2). Os resultados apresentados na data de 7/12 da Tabela 2 são referentes a um exame realizado após 45 dias da alta hospitalar, sendo possível verificar 
que os valores das enzimas séricas AST, GGT e CPK ainda permaneciam acima dos parâmetros normais. Observou-se melhora do quadro clínico, resposta positiva ao tratamento e o paciente recebeu alta. A ureia e a creatinina seguiram com valores aumentados, o que indica possível falha renal devido ao efeito deletério da hemoglobina liberada, que é eliminada pelos rins levando a uma nefrose aguda (RIBEIRO, 2011). De acordo com Câmara et al. (2016), após a crise hemolítica o Cu, os radicais livres, as lisozimas e hemoglobina são filtradas pelos rins provocando quadro lesivo nos glomérulos e túbulos renais, resultando em insuficiência renal, sendo esta, possivelmente, a causa da elevação dos valores de ureia e creatinina. 


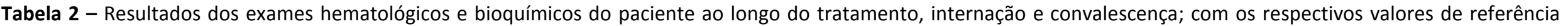
para a espécie ovina.

\begin{tabular}{|c|c|c|c|c|c|c|c|c|c|c|c|c|c|c|}
\hline $\begin{array}{l}\text { Data da coleta } \\
\text { HEMOGRAMA }\end{array}$ & $21 / 9$ & $22 / 9$ & $23 / 9$ & $24 / 9$ & $25 / 9$ & $28 / 9$ & $30 / 9$ & $5 / 10$ & $7 / 10$ & $13 / 10$ & $19 / 10$ & $22 / 10$ & $7 / 12$ & $\begin{array}{l}\text { Valores de } \\
\text { Referência* }\end{array}$ \\
\hline \multicolumn{15}{|l|}{ Eritrograma } \\
\hline Eritrócitos $\left(\times 10^{6 /} \mu \mathrm{L}\right)$ & 4.85 & 4.66 & 3.51 & 1.97 & 3.81 & 2.97 & 2.09 & 3.36 & 3.16 & 3.17 & 5.51 & & 10.3 & 9 a 15 \\
\hline Hemoglobina (g/dL) & 7.2 & 6.6 & 5.8 & 5.2 & 5.7 & 4.9 & 4.5 & 6.1 & 4.9 & 5.1 & 7.3 & & 11.2 & 9 a 15 \\
\hline Hematócrito (\%) & 19 & 19 & 16 & 15 & 16 & 15 & 14 & 17 & 15 & 16 & 20 & & 33 & 27 a 45 \\
\hline V.C.M. (fL) & 39.2 & 47.5 & 45.6 & 150.0 & 41.99 & 50.38 & 66.98 & 56.7 & 47.5 & 50.47 & 36.29 & & 32.9 & 28 a 40 \\
\hline C.H.C.M. (\%) & 37.9 & 31.6 & 36.3 & 33.3 & 35.62 & 32.66 & 32.14 & 35.3 & 32.7 & 31.87 & 36.50 & & 33.93 & 31 a 34 \\
\hline Policromasia & & $1+$ & & & & & $1+$ & & & & $1+$ & & & \\
\hline Anisocitose & & $1+$ & & $1+$ & $2+$ & $1+$ & $1+$ & & & & $3+$ & & & \\
\hline Proteína Plasmática Total (g/L) & 76 & 70 & 66 & 64 & 60 & 60 & 58 & 90 & 94 & 94 & 90 & 91 & 82 & 60 a 79 \\
\hline \multicolumn{15}{|l|}{ Leucograma } \\
\hline Leucócitos totais $(/ \mu \mathrm{L})$ & 10447 & 4900 & 4600 & 5100 & 5500 & 7500 & 9200 & 14300 & 11900 & 9200 & 7700 & & 7500 & 4000 a 12000 \\
\hline N. Segmentados $(/ \mu \mathrm{L})$ & 3865 & 1862 & 2714 & 2550 & 3300 & 3375 & 6072 & 10582 & 8211 & 5336 & 2464 & & 2775 & 699 a 6000 \\
\hline Eosinófilos $(/ \mu \mathrm{L})$ & 209 & 343 & 0 & 0 & 55 & 0 & 0 & 0 & 0 & 0 & 77 & & 150 & 1 a 1000 \\
\hline Basófilos $(/ \mu \mathrm{L})$ & 0 & 0 & 0 & 0 & 0 & 0 & 0 & 0 & 0 & 0 & 0 & & 0 & 1 a 300 \\
\hline Monócitos $(/ \mu \mathrm{L})$ & 0 & 196 & 0 & 255 & 55 & 450 & 92 & 429 & 357 & 92 & 154 & & 375 & 1 a 750 \\
\hline Linfócitos $(/ \mu \mathrm{L})$ & 6373 & 2499 & 1886 & 2295 & 2090 & 3675 & 3036 & 3289 & 3332 & 3772 & 5005 & & 4200 & 2000 a 9000 \\
\hline \multicolumn{15}{|l|}{ BIOQUÍMICOS } \\
\hline TGO (AST) (U/L) & 1260.0 & 1392.0 & & & & & & & 161 & & & 222.0 & 156 & 0 a 90 \\
\hline CPK (U/L) & 3018.0 & 2341.0 & & & & & & & 104 & & & 313 & 96.0 & 0 a 40 \\
\hline Creatinina (mg/dL) & 6.74 & 7.95 & 9,26 & 10.01 & 11.15 & 13.86 & 14.81 & 14.45 & 13.71 & & 4.05 & 3.33 & 2.4 & 1,2 a 1,9 \\
\hline Uréia (mg/dL) & 329.0 & 429.0 & 506 & 447.0 & 520 & 508 & 488 & 533.0 & 543 & & 174 & 173.0 & 57.9 & 17 a 43 \\
\hline GGT (U/L) & & & & 278.0 & & & & & & & & & 148.0 & 0 a 32 \\
\hline
\end{tabular}

*Jain (1993), Kaneko et al. (1997). 
A intoxicação por Cu pode ser irreversível se for diagnosticada em fase avançada, portanto o tratamento torna-se eficiente se for iniciado precocemente. A taxa de mortalidade da doença é alta. Segundo Ferreira et al. (2008), o coeficiente de letalidade depende da realização do tratamento e da intervenção no momento oportuno, a possibilidade de morte chega a 95\% nos animais não tratados. A maioria dos casos de ICA descritos na literatura apresentam diagnósticos firmados através de achados necroscópicos, visto que os animais evoluem para óbito. Ribeiro (2011) descreve achados como fígado ictérico, rins hemorrágicos e escuros, líquido na cavidade abdominal e torácica. Os exames de imagem como a ultrassonografia podem ser úteis para investigar o acúmulo de líquido na cavidade abdominal e torácica, rins tumefeitos, fígado congesto e com tamanho aumentado. Bandinelli et al. (2013), relata alterações na necropsia como hepatomegalia, erosões e ulcerações multifocais aleatórias na mucosa abomasal. Na avaliação histológica relata necrose hepatocelular centrolobular acentuada e difusa tumefação de hepatócitos. Alterações macroscópicas como icterícia generalizada da carcaça, rins com tumefação bilateral do parênquima renal e bexiga contendo urina de coloração enegrecida, são apontadas por Câmara et al. (2016).

No presente relato, o sucesso da terapia permitiu que o carneiro voltasse a ser utilizado como reprodutor da Cabanha, com relação custo/benefício favorável, tendo em vista o valor genético do reprodutor e a venda futura da sua progênie. No RS, este tema se torna relevante frente ao crescimento expressivo da ovinocultura, da intensificação dos sistemas de produção e o desconhecimento, por parte de grande parcela dos produtores, quanto ao correto manejo nutricional dos pequenos ruminantes. Pois, de modo geral, utilizam alimentos concentrados em excesso para que os animais expressem seu máximo potencial produtivo, predispondo-os a doenças como ICA, urolitíase e acidose ruminal (RIBEIRO, 2011). O presente trabalho representa uma contribuição à Medicina Veterinária, visto que a maioria dos casos descritos considera o tratamento oneroso, prolongado e, portanto, não usual e recomendado. Geralmente os animais vão a óbito, sendo o diagnóstico confirmado através de exames post mortem. Esse relato tem impacto no que tange ao conhecimento do 
tratamento, recuperação e diagnóstico dos casos de intoxicação cúprica acumulativa em ovinos.

\title{
CONCLUSÃO
}

A pronta instituição da terapia, de modo intensivo, em ambiente hospitalar, foi fundamental para a recuperação do quadro clínico. O diagnóstico de intoxicação cúprica acumulativa foi baseado no histórico, exame clínico, análises laboratoriais e no resultado do tratamento, porém, em novos casos, se recomenda a realização de biópsia hepática para obter um diagnóstico definitivo e conclusivo.

\section{CUMULATIVE CUPRIC POISONING IN A SHEEP: TREATMENT AND RECOVERY (CASE REPORT)}

\section{ABSTRACT}

\begin{abstract}
C opper intoxication is one of the leading causes of intoxication mortality in sheep. The clinical and anatomopathological signs are similar to those of other diseases. Thus, several cases become inconclusive when analytical techniques are not used in the diagnosis. This study reports the case of a Hampshire Dow ram that was transferred to the Veterinary Clinics Hospital of the Federal University of Rio Grande do Sul with clinical signs of copper intoxication. The animal showed apathy, weight and appetite loss, dyspnea, cough, nasal secretion, and black colored urine and diarrhea. The ram was purchased at an animal exhibition fair and had remained in intensive management receiving amounts of concentrate over the dietary requirement two months prior and during the event. Four days after the appearance of clinical signs the animal was admitted to the hospital and support therapy was established, with fluid therapy, amino acids and glucose supplementation. Sodium sulfate, ammonium molybdate, toxin adsorbent and mucosal protectors were administered. After 34 days of intensive treatment, the patient showed improvement of the clinical condition and was discharged. Based on historic, species sensitivity, clinical signs, results of laboratory tests, improvement of the clinical condition and positive response to therapeutics, it is suggested the clinical condition of cumulative cupric poisoning. No specific histochemical analysis for copper marking was performed. The study consists of literary review, clinical and laboratory characteristics of intoxication, conduct for treatment and diagnosis, contributing, thus, to the understanding of the disease and reducing new cases occurrence.
\end{abstract}

Keywords: Copper. Hemoglobinuria. Hemolysis. Diagnosis. 


\section{INTOXICACIÓN CÚPRICA ACUMULATIVA EM OVINO: TRATAMIENTO Y RECUPERACIÓN (RELATO DE CASO)}

\section{RESUMEN}

a intoxicación por cobre es una de las principales causas de mortalidad por intoxicación en ovinos. Los signos clínicos y anatomopatológicos son similares a los de otras enfermedades. Así, varios casos se vuelven inconclusos cuando no se utilizan técnicas analíticas en el diagnóstico. El presente trabajo relata el caso de un carnero de la raza Hampshire Dow que fue encaminado al Hospital de Clínicas Veterinarias de la Universidad Federal de Rio Grande do Sul con signos de intoxicación por cobre. El animal presentaba apatía, pérdida de apetito, adelgazamiento, disnea, tos, secreción nasal, orina y diarrea de coloración oscura. El carnero fue adquirido en una feria de exposición de animales y había permanecido con un manejo intensivo recibiendo cantidades de concentrado por encima de la exigencia dietética, en los dos meses previos y durante el evento. Cuatro días después del inicio de los signos clínicos el animal fue ingresado en el hospital y se estableció terapia de soporte con fluidoterapia, suplementación de aminoácidos y glucosa. Se administró sulfato de sodio, molibdato de amonio, adsorbente de toxinas y protectores de la mucosa. Después de 34 días de tratamiento intensivo el paciente presentó mejoría del cuadro clínico y fue dado de alta. En base al historial, la sensibilidad de la especie, signos clínicos, los resultados de los exámenes de laboratorio, mejora del cuadro clínico y la respuesta positiva a la terapéutica, se sugiere el cuadro de intoxicación acumulativa cúprica. No se realizó un análisis histoquímico específico para la detección de cobre. El trabajo consiste en revisión literaria, características clínicas y de laboratorio de la intoxicación, conducta para el tratamiento y diagnóstico, contribuyendo, por lo tanto, para el entendimiento de la enfermedad y reducción de la ocurrencia de nuevos casos.

Palabras clave: Cobre. Hemoglobinuria. Hemólisis. Diagnóstico.

\section{REFERÊNCIAS}

ALMEIDA, T. L.; BRUM, K. B.; LEMOS, R. A. A.; LEAL, C. R. B.; BORGES, F. A. Doenças de ovinos diagnosticadas no Laboratório de Anatomia Patológica Animal da Universidade Federal de Mato Grosso do Sul (1996-2010). Pesquisa Veterinária Brasileira, v. 33, n. 1, p. 21-29, 2013.

ANTONELLI, A. C.; WANDERLEY, C. W. S; ORTOLANI, E. L. Intoxicação cúprica acumulativa em ovinos: Revisão de literatura. PUBVET, Londrina, v. 4, n. 34, p. 938, 2010. 
BANDINELLI, M. B.; PAVARINI, S. P.; GOMES, D. C.; et al. Intoxicação aguda por cobre em ovinos. Ciência Rural, v. 43, n. 10, p. 1862-1865, 2013.

CÂMARA, A. C. L.; OLINDA, R. G.; DALCIN, L.; et al. Intoxicação cúprica acumulativa em ovinos no Nordeste brasileiro. Acta Scientiae Veterinariae, Porto Alegre, v. 44, n. 1, p. 1-6, 2016.

CORREA, G. L. F. Estudo Retrospectivo das Causas de Morte de Ovinos Diagnosticados no Setor de Patologia Veterinária - UFRGS: 2002-2012. Porto Alegre: UFRGS, 2014. 50p. Dissertação (Mestrado em Ciências Veterinárias), Faculdade de Veterinária, Universidade Federal do Rio Grande do Sul, 2014.

COSTA, T. N. Intoxicação por cobre aspectos clínicos e laboratoriais. Goiânia: UFG, 2011. 27p. Disciplina de Seminários Aplicados, Programa de Pós-Graduação em Ciência Animal, Escola de Veterinária e Zootecnia, Universidade Federal de Goiás, 2011. Disponível em: < https://portais.ufg.br/up/67/o/semi2011_Thays_Nascimento_2.pdf>.

FERREIRA, D. O. L. Modelo experimental de urolitíase em ovinos - estudo clínico, laboratorial e hemogasométrico. Botucatu: UNESP, 2013. 219p. Tese (Doutorado), Programa de Pós-Graduação em Medicina Veterinária, Faculdade de Medicina Veterinária e Zootecnia, Universidade Estadual Paulista, 2013.

FERREIRA, M. B.; ANTONELLI, A. C.; ORTOLANI, E. L. Intoxicação por cobre, selênio, zinco e cloreto de sódio. In: SPINOSA, H. S.; GÓRNIAK, S. L.; NETO, J. P. Toxicologia aplicada à medicina veterinária. Barueri: Editora Manole, 2008. 960p.

GONZÁLEZ, F. H.; SILVA, S. C. Introdução à Bioquímica Clínica Veterinária. 2. ed. Porto Alegre: UFRGS, 2006. Cap.6, p. 211-250.

JAIN, N. C. Essentials of veterinary hematology. Philadelphia: Lea \& Febiger, 1993. 417p.

KANEKO, J. J.; HARVEY, J. W.; BRUSS, M. L. (Eds.) Clinical biochemistry of domestic animals. 5. ed. New York: Academic Press, 1997. 932p.

MIGUEL, M. P.; SOUZA, M. A.; CUNHA, P. H. J.; et al. Intoxicação crônica por cobre em ovinos: conduta para o diagnóstico conclusivo. Arquivo Brasileiro de Medicina Veterinária e Zootecnia, v. 65, n. 2, p. 364-368. 2013.

MÖLLERKE, R. O.; RIBEIRO, L. A. O. Aspectos epidemiológicos da intoxicação crônica por cobre em ovinos preparados para exposição. Veterinária em Foco, Porto Alegre, v. 2, n. 2, p. 183-192, 2005.

NETO, H. M. A.; FREITAS, M. D.; SANCHEZ, I. X. B. Considerações sobre intoxicação crônica por cobre em ovinos. Revista Colombiana de Ciência Animal, v. 7, n. 1, p. 98-107, 2014. 
OSWEILER, G. D. Toxicologia Veterinária. Porto Alegre: Artes Médicas, 1998. Cap. 17, p. 212215.

PUGH, D. G. Clínica de Ovinos e Caprinos. São Paulo: Roca, 2005. Cap. 10, p. 287-310.

RADOSTITS, O. M.; GAY, C. C.; BLOOD, D. C.; et al. Clínica Veterinária Um Tratado de Doenças dos Bovinos, Ovinos, Suínos, Caprinos e Equinos. 9. ed. Rio de Janeiro: Guanabara Koogan, 2000. Cap. 31, p. 1416-1471.

RIET-CORREA, F.; SCHILD, A. L.; MÉNDEZ, M. D. C.; et al. Doenças de Ruminantes e Equinos. 2. ed. São Paulo: Varela, 2001. Cap. 2, p. 163-217.

REIS, M. O.; MELLO, L. S.; CRUZ, R. A. S.; et al. Intoxicação crônica por cobre em ovinos alimentados com bagaço de uva. Acta Scientiae Veterinariae, Porto Alegre, v. 43, n. 1, p. 108, 2015.

RIBEIRO, L. A. O. Medicina de Ovinos. Porto Alegre: Pacartes, 2011. P. 177-190.

RIBEIRO, L. A. O.; RODRIGUES, N. C.; SMIDERLE, W. A. Intoxicação crônica por cobre em ovelhas pastoreadas em pomar de videiras adubado com cama aviária. Brazilian Journal of Veterinary Research and Animal Science, São Paulo, v. 44, n. 3, p. 208-211, 2007.

SMITH, B. P. Medicina Interna de Grandes Animales. 4. ed. Espanha: Elsevier, 2010. Cap. 35, p. 1039-1084. 